\title{
Smart Home Security System using lot, Face Recognition and Raspberry Pi
}

\author{
Manoj R. Dhobale \\ Dept. of Computer Engineering \\ V.V.P. Polytechnic, Solapur, \\ Maharashtra, India
}

\author{
Rekha Y. Biradar \\ Dept. of Computer Engineering \\ V.V.P. Polytechnic, Solapur, \\ Maharashtra, India \\ Sharad A. Awatade \\ Dept. of Computer Engineering \\ V.V.P. Polytechnic, Solapur, \\ Maharashtra, India
}

\author{
Raju R. Pawar \\ Dept. of Computer Engineering \\ V.V.P. Polytechnic, Solapur, \\ Maharashtra, India
}

\begin{abstract}
In the today's world the security of the home is the prime concern. The traditional methods of securing our home are very easy to break and lead to theft. To protect the home, we need to install the costly security system. To overcome this problem, we are presenting IoT based solution where we can setup a smart home security system. In this paper we are proposing the system with the help of face recognition to develop the automatic door lock and unlock system. It also gives us the facility to monitor our home remotely and take appropriate action if anything goes wrong. The Pi camera will be attached to the Raspberry pi accompanied with Passive Infrared and other sensors. Camera captures an image of the person in front of the door, then real-time face recognition is done using local binary pattern (LBP) [3]. If person's image matches with one of the home members or identified person then the door will unlock otherwise won't. Email containing image of the unknown person will be sent to the homeowner to his Gmail Id. The proposed system keeps the owner informed in the real time about the unknown person at the door of the home.This information will help the user to take necessary actions.
\end{abstract}

\section{General Terms}

Home Security System

\section{Keywords}

Home Security System, Face Recognition, Raspberry Pi

\section{INTRODUCTION}

A home security is the prime concern to anybody. A home security system is required to keep our belongings and valuables safe from any kind of robbery or theft. In the United States, there is a home related burglary that takes place every 13 seconds, 4 burglaries a minute, 240 an hour and nearly 6,000 a day! some of the statistics are $88 \%$ of all burglaries are residential in nature, $77 \%$ of all crimes are property crimes, $38 \%$ of all robberies are committed with guns, identity theft is the fastest growing crime in the U.S., Canada and UK. 3 out of 4 homes in the U.S. Will be broken within the next 20 years [5]. The traditional home security system is easily breakable and quite outdated. This in turns results in the robbery and also needs installation of the costly security system. Our proposed system delivers a cost and energy efficient solution for home security by using IoT and face recognition. LBP based Face recognition system help us to detect the person at the door with more accuracy.[Shown in Fig1.a and Fig1.b][9]The internet of things, or IoT, is a system of interrelated computing devices, mechanical and digital machines, objects, animals or people that are provided with unique identifiers (UIDs) and the ability to transfer data over a network without requiring human-to-human or humanto-computer interaction.[6] Data transmission over a network can be made part of IoT system by embedding them with electronic hardware such as sensors, software and networking gear.

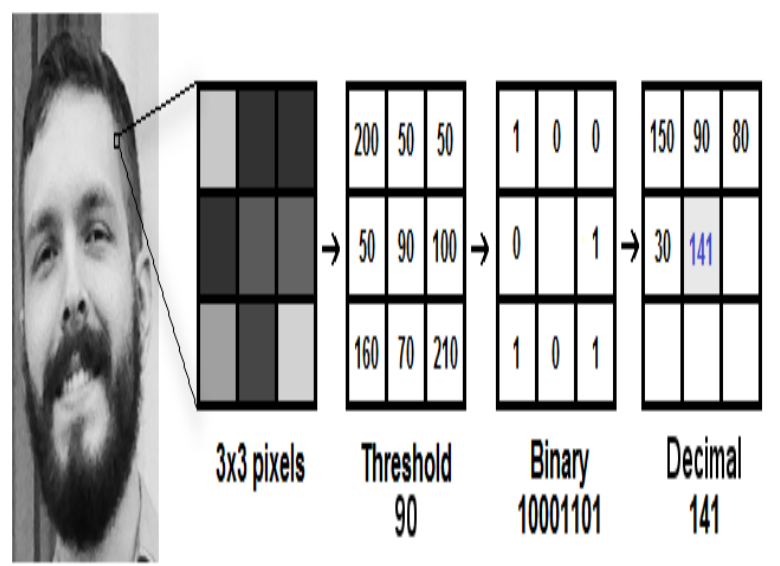

Fig .1.a LBP feature extraction procedure[9]

\section{LITERATURE REVIEW}

The latest technologies which are available for security includes RFID card technologies, biometric, OTP based unlock system and many more. Each of which is effective in some areas but don't provide the complete security system. Many of the researchers have proposed home security system but very few used face recognitions for it. Face recognition has the good potential to offer a strong security system to the home. [7]. A Face Recognition System Based on Local Binary Patterns and Support Vector Machine for Home Security Service Robot by authors Jia Kailin Wang, Jinjin Zheng, Shiwu Zhang, Jijun $\mathrm{He}$, Xiao Liang and Sui Feng has explained how LBP works in face detection module but lack the support in case of power failure[8]. 


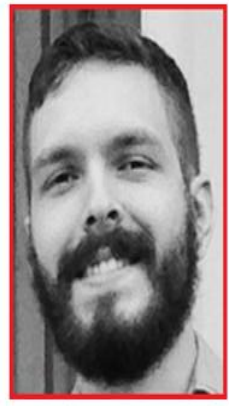

Original Image

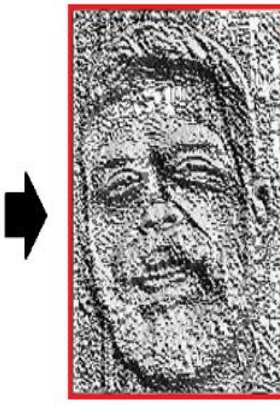

LBP Result

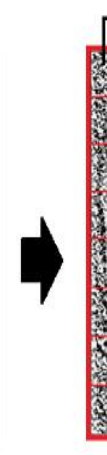

Regions/Grids

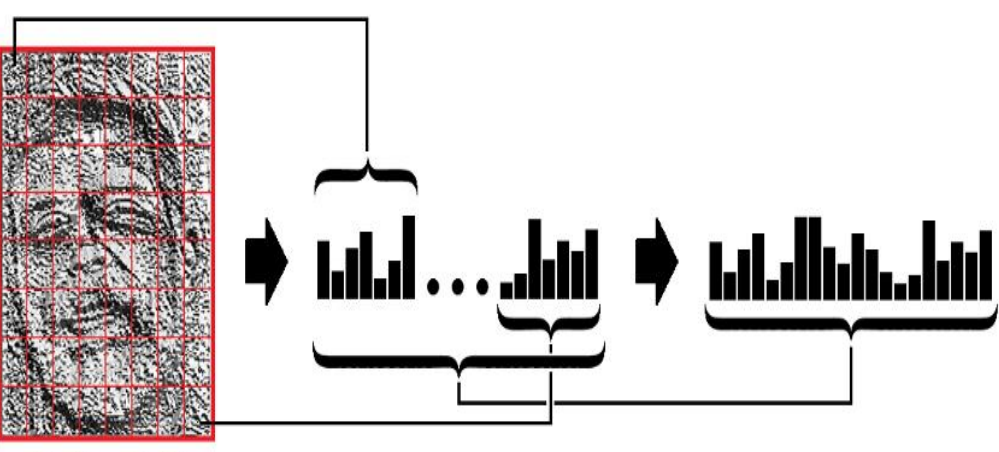

Histogram of each region

Concatenated Histogram

Fig .1.b LBP extraction procedure [9]

In [2], Face Recognition System Using IoT by authors Sandesh Kulkarni,Minakshee Bagul, Akansha Dukare, Prof. Archana Gaikwad, has the proposed system very well presented. The methodology is quite well explained in detail. The working of door lock-unlock on face recognition is very good, but use of Wi-Fi limits its area of coverage and home owner cannot access the system remotely. Another disadvantages of above systems that they do not inform the owner regarding the security breach issue on their Smartphone so that he can take the appropriate action on it.

\section{METHODOLOGY}

\subsection{Proposed System}

Our proposed system consists of raspberry pi as a main controller which is cost effective and requires very less power to operate. It has embedded sensor like PIR for motion detection and ultrasonic sensors to measure the distance. The stepper motor is used to help the door lock-unlock mechanism which is controlled by Raspberry pi. Block diagram of proposed system is shown in fig2.

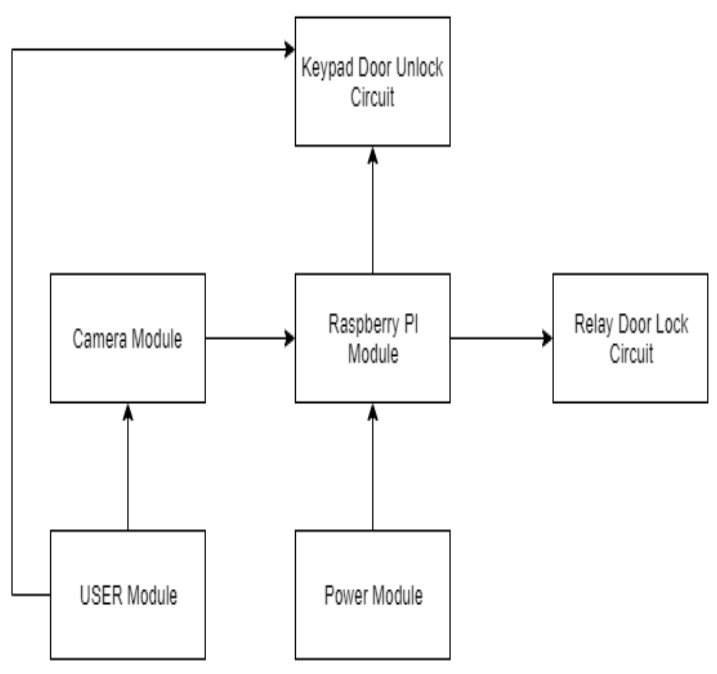

Fig.2 The block diagram of the proposed system:

The Raspberry pi consists of a face recognition algorithm, web application and an Android system. In our proposed system we have used the cloud to send the data to the remote Smartphone which is at the user side. This is the IoT based system and can be made automated as and when required. It has very low power requirement which makes it most affordable. It requires minimum $5 \mathrm{~V}$ to work. We can easily replace any part of this system without disturbing the other parts. The system will consist of the identified member's photos in the database. Any new entry can be made as and when required in the database. Image will be captured and face recognition will be performed. If face match found the door will open else red color bulb will light up, if the owner is at home(specified in android application) then bell will ring else notification will be sent i.e., someone is waiting outside your home. An email will be sent to the owner on his Gmail along with the photo of the unknown

person if the person is not identified. All these activities are stored in the database which is in micro SD card in raspberry pi and maximum size of SD card is 32GB. All the data is available on the Internet which makes our system more efficient.

\subsection{Local Binary Pattern}

Texture classification is needed for face recognition. For this Local Binary Pattern (LBP) approach is used. LBP is a simple and very efficient texture operator which labels the pixels of an image by thresholding the neighborhood of each pixel and considers the result as a binary number.It was first described in 1994 (LBP) and has since been found to be a powerful feature for texture classification. It has further been determined that when LBP is combined with histograms of oriented gradients (HOG) descriptor, it improves the detection performance considerably on some datasets. LBP computes local representation of texture and it is computed by comparing each pixel with its surrounding neighborhood of pixel. For texture descriptor, image needs to be converted into gray scale image.Using the LBP combined with histograms, the face images are represented with a simple data vector [9].

\subsection{Raspberry Pi}

The Raspberry Pi is a very cheap computer that runs Linux, but it also provides a set of GPIO (general purpose input/output) pins that allow you to control electronic components for physical computing and explore the Internet of Things (IoT). Raspberry Pi GPIO is shown in figure 3.All over the world, people use Raspberry Pi to learn programming skills,build hardware projects, do home automation, and even use them in industrial applications. In proposed system, GPIO pins are controlled using python programming. Any of the GPIO pin is designed as input or output using available software [10]. Raspberry pi operates in the open source ecosystem. Its main supported operating system is Raspbian and it is open source. 


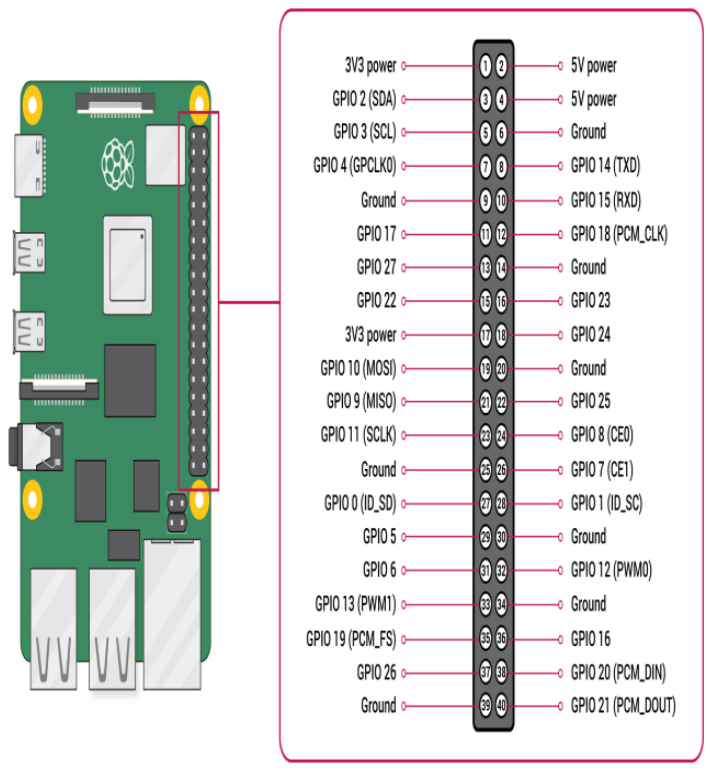

Fig. 3 Raspberry pi GPIO layout

\section{RESULT}

After capturing the photo at the door face recognition is done to check whether person is a home member or identified one. It will determine if the person is stranger or not. If the person is stranger the led will turn red and the door will not open. If the person is a stranger then his photo will be sent to the owners Gmail ID, the photo is shown in the figure 4. Working model of system is shown in figure 5 .

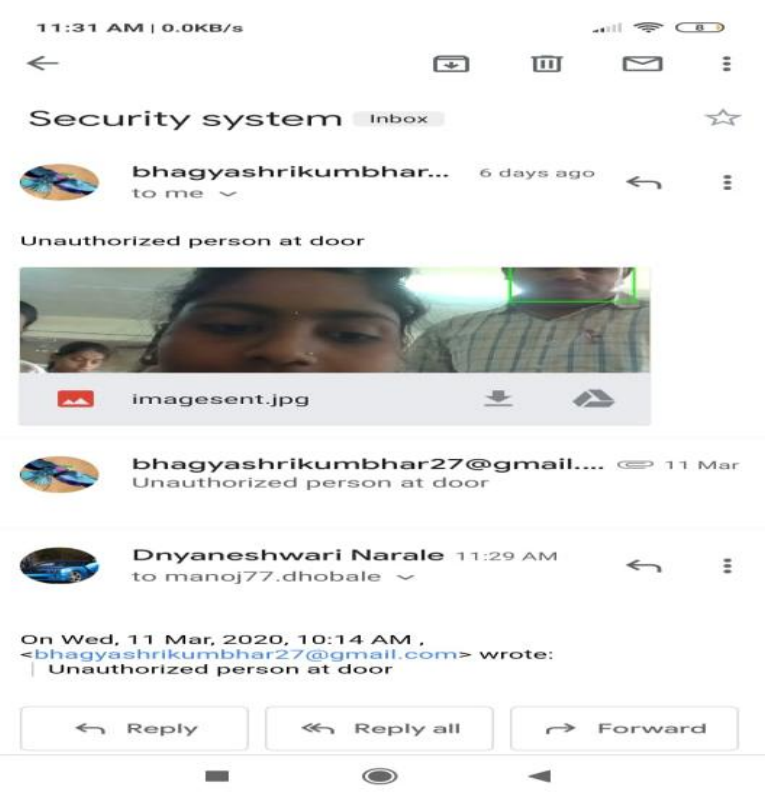

Fig. 4 Unknown person identified at the door

\section{FUTURE SCOPE}

In the future a more accurate and exact face recognition algorithm can be presented. We can control the access to the door using our Smartphone remotely by sending the signals to the system. We can build an Android application to control our system more easily and efficiently. We can even provide live streaming of the video to the owner on his Smartphone.

\section{CONCLUSION}

In this paper we have proposed and demonstrated smart home security approach which requires less cost and provide the security to our home. We have the system withthe help IoT and face recognition. The real time face recognition allows us to detect the stranger atthe door and give notification to the owner on his Gmail regarding this.

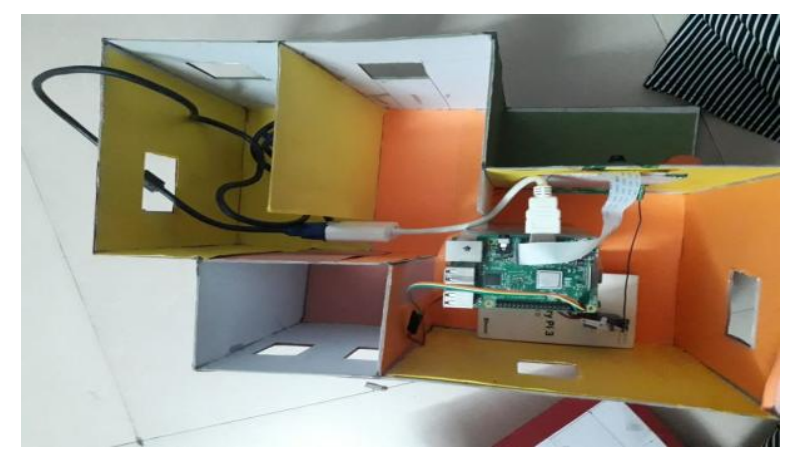

Fig. 5 Working model of the proposed system with Raspberri Pi 3

\section{ACKNOWLEDGMENTS}

Authors would like to thank Prof. Shaikh SM., Principal, V.V.P. Polytechnic, Solapur, for his constant guidance and family members for their unconditional love and support.

\section{REFERENCES}

[1] IndiaBurglaryRate,20032016:https://knoema.com/atlas/I ndia/topics/Crime-Statistics/Burglary-Car-Theft-andHousebreaking/Burglary-ra te

[2] Sandesh Kulkarni, Minakshee Bagul, AkanshaDukare, Prof. Archana Gaikwad. "Face Recognition System Using IoT", International Journal ofAdvanced Research in Computer Engineering\&Technology, November-2017.

[3] Zhengzheng Liu, Lianrong Lv, "Development of face Recognition System Based on PCA and LBP for Intelligent Anti- Theft Doors", 2nd IEEE International Conference on Computer and Communications,2016.

[4] Anjali Patel, "IoT based Facial door access control home security system", "International Journal of Computer Applications, August 2017.

[5] HomeBurglary Statistics 2019: https://reolink.com/homeburglary-crime-statistics/

[6] Internet of Things-IoT Agenda-TechTarget: https://internetofthingsagenda.techtarget.com/definition/I nternet-of-Things-IoT

[7] Dwi Ana Ratna, Wati Dika Abadianto, "Design of Face Detection and Recognition System for Smart Home Security Application", $20172^{\text {nd }}$ ICITISEE

[8] Jiakailin Wang, Jinjin Zheng, Shiwu Zhang, Jijun He, Xiao Liang and Sui Feng, "A Face Recognition System Based on Local Binary Patterns and Support Vector Machine for Home Security Service Robot”, 2016 9th International Symposium on Computational Intelligence and Design

[9] LocalBinaryPattern:https://towardsdatascience.com/facerecognition-how-lbph-works-90ec258c3d6b

[10] Raspberry Pi: www.raspberrypi.org 